American Journal of Applied Sciences 9 (8): 1225-1231, 2012

ISSN 1546-9239

(C) 2012 Science Publications

\title{
Effects of Gas Velocity and Temperature on Nitric Oxide Conversion in Simulated Catalytic Converter
}

\author{
Sathaporn Chuepeng \\ Department of Mechanical Engineering, Faculty of Engineering at Si Racha, \\ Kasetsart University, 199 Sukhumvit Road, Chonburi 20230, Thailand
}

\begin{abstract}
Problem statement: Gaseous emissions from gasoline engine such as carbon monoxide, unburned hydrocarbon and nitrogen oxides were usually reduced in three-way catalytic converter simultaneously around theoretical fuel and air combustion. Engine speed and load and other parameters were varied over a wide range of operating conditions, resulting in different exhaust gas composition and condition intake into catalytic converter. This work was studied the conversion of Nitric Oxide (NO) in exhaust gas catalytic converter affected by gas velocity and inlet temperature using numerical modeling. Approach: The simulation was based on a one-dimensional timedependent model within a single monolith channel of the converter. Upon certain assumptions, the study was considered heterogeneous combustion reaction between gas and solid phases based on lumped kinetic reactions. In this study, constants and variables used for mass and heat transfers were dependent on gas or solid phase temperature and mole fraction. Finite difference scheme incorporated with the generated computer code was established for solving species and energy balances within gas and solid phases. Results: The NO conversion was increased with transient period in initial and reached steady state at different values. The lower inlet gas temperature was resulted in lesser NO conversion at the same inlet NO concentration and gas velocity. The light-off temperatures were up to $520 \mathrm{~K}$ and a sudden rise in $\mathrm{NO}$ conversion was from 550-605 K and decreasing onwards, generating working temperature window. NO conversion increased throughout the catalyst bed from the inlet and the conversion decreased as the gas velocity increased. Conclusion/Recommendations: Gas space velocity and gas temperature intake to the converter affected the NO conversion over the time and the axial distance from the catalyst bed inlet. The numerical results have summarily demonstrated a good approximation compared to experimental data provided in the literature. Further investigation of such effects on other gaseous components is recommended for future work.
\end{abstract}

Key words: Nitric Oxide (NO), unburned Hydrocarbon (HC), Carbon Monoxide (CO), numerical simulation, catalytic converter, heterogeneous combustion, finite difference method, conversion, NO conversion

\section{INTRODUCTION}

Three-way catalytic converter for gasoline engine usually reduces regulated gaseous emissions (i.e., carbon monoxide, unburned hydrocarbon and nitrogen oxides) simultaneously in the range about stoichiometric fuel and air combustion. However, engine parameters are varied over a wide range of operating conditions, e.g., speed, load, resulting in different conditions of exhaust gas flowing into catalytic converter. The consequences are dissimilarity in conversion of these harmful gaseous emissions into usual combustion products, particularly Nitric Oxide (NO, a major constituent in nitrogen oxides, $\mathrm{NO}_{\mathrm{x}}$, emissions) with their high level adjacent to stoichiometric combustion. A number of experimental studies associated to the reduction of exhaust gas emissions in catalytic converter were conducted (Botas et al., 2001). For such engines operating in fuel lean condition, e.g., gasoline direct injection, a conversion of nitrogen oxides in oxidizing atmosphere is even more complicated (Holma et al., 2004) and dependent on type of catalyst/washcoat/substrate (Furusawa et al., 2002). These bring complication of both physical and chemical parameters which need to overcome. Numerical simulation is therefore an alternative way for solving these difficulties (Hepburn et al., 1998).

Kolaczkowski (1999) presented a catalytic combustion modeling in monolith reactors. Tischer et al. (2001) simulated a catalytic combustion using detailed models for heterogeneous and homogeneous reactions and transport phenomena. These models crucially require kinetic rates of reactions taken place. A number of studies on kinetic rates of specific 
reactions and combustion conditions over particular catalyst/washcoat have been proposed. Voltz et al. (1973) studied the carbon monoxide and propylene oxidation on platinum catalysts and proposed their kinetic reaction rates. Under fuel-lean combustion conditions, Burch and Watling (1997) studied kinetics and mechanism of the NO reduction by propane over platinum catalyst.

From engineering point of view, it is interest to conduct such integration of the aforementioned catalytic combustion modeling and kinetic reaction rates. The main aim of this study is first to present a one-dimensional transient modeling in monolith catalytic converter. Secondly, NO emission conversion will be studied through the modeling. Some operating parameters, i.e., inlet gas temperature, bed length and gas velocity affected on NO conversion will be simulated and discussed.

\section{MATERIALS AND METHODS}

Catalytic converter modeling: The modeling of catalytic converter with the $0.16 \mathrm{~m}$ catalyst bed length shown in Fig. 1 was based on one-dimensional transient model. Exhaust gases as combustion products, in the form of mole fraction at given temperature, from engine flowing to the inlet of catalyst bed. The cross-sectional area of a single monolith channel is depicted in Fig. 2. The catalyst and washcoat are solid phase as illustrated by the shade area.

Heterogeneous combustion: Heterogeneous combustion occurred between gas phase (exhaust gas species) and solid phase (catalyst/washcoat) is upon the following assumptions:

- No homogeneous reaction between species in gas phase

- Neglecting transient term in gas phase

- Mass and energy transport in gas phase only by convection

- Energy transport in solid phase only by conduction and

- Neglecting radiation heat transfer

It is considered in this study that the exhaust gas comprises the combustion products: Carbon Dioxide $\left(\mathrm{CO}_{2}\right)$, Water Vapor $\left(\mathrm{H}_{2} \mathrm{O}\right)$, Nitrogen $\left(\mathrm{N}_{2}\right)$, unburned Hydrocarbon (HC), Carbon Monoxide (CO), Nitric Oxide (NO) and excess Oxygen $\left(\mathrm{O}_{2}\right)$. Species and energy balances were used for the analysis.

Species equations: For species balance, each species in the form of mole fraction flowed in gas phase without chemical reaction and mass can diffuse from the gas phase to solid phase.

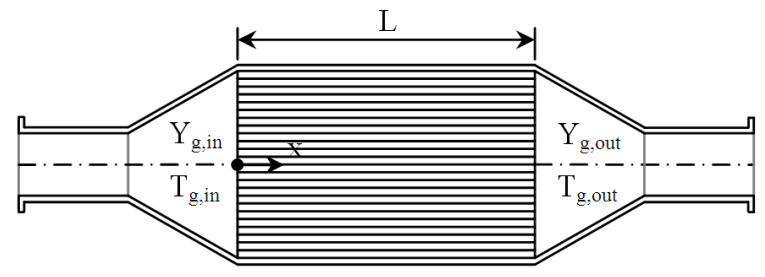

Fig. 1: Parameters of the one-dimensional catalytic converter model with $0.16-\mathrm{m}$ catalyst bed Length (L)

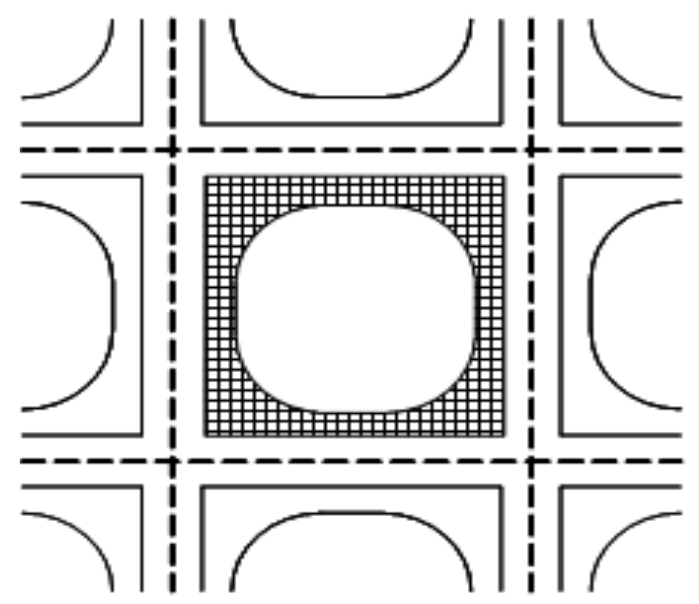

Fig. 2: A single monolith channel of the model (Hayes and Kolaczkowski, 1997)

In the solid phase, there was a chemical reaction and mass can also diffuse from the solid phase to the gas phase. The species balance in the gas and solid phases, originally given by Hayes and Kolaczkowski (1997) and applied here for this problem, can be expressed by Eq. 1 and 2, respectively:

$$
\begin{aligned}
& \mathrm{C}_{\mathrm{g}} \mathrm{v}_{\mathrm{m}}^{*} \frac{\mathrm{dY} \mathrm{Y}_{\mathrm{A}, \mathrm{g}}}{\mathrm{dx}}+\frac{4}{\mathrm{D}_{\mathrm{H}}} \mathrm{k}_{\mathrm{m}, \mathrm{A}} \mathrm{C}_{\mathrm{g}}\left(\mathrm{Y}_{\mathrm{A}, \mathrm{g}}-\mathrm{Y}_{\mathrm{A}, \mathrm{s}}\right)=0 \\
& \mathrm{k}_{\mathrm{m}, \mathrm{A}} \mathrm{C}_{\mathrm{g}}\left(\mathrm{Y}_{\mathrm{A}, \mathrm{g}}-\mathrm{Y}_{\mathrm{A}, \mathrm{s}}\right)=\sum_{\mathrm{i}} \mathrm{v}_{\mathrm{Ai}} \mathrm{R}_{\mathrm{i}, \mathrm{s}}
\end{aligned}
$$

Where:

$$
\begin{array}{ll}
\mathrm{C}_{\mathrm{g}} & =\text { Gas concentration in mol } \mathrm{m}^{-3} \\
\mathrm{v}_{\mathrm{m}}^{*} & \text { Mean mole average velocity in } \mathrm{m} \\
& \sec ^{-1} \\
= & \text { Hydraulic diameter in } \mathrm{m} \\
\mathrm{D}_{\mathrm{H}} & \text { Mass transfer coefficient of species } \mathrm{A} \\
\mathrm{k}_{\mathrm{m} . \mathrm{A}} & \text { in } \mathrm{m} \mathrm{sec}^{-1} \\
\mathrm{Y}_{\mathrm{A}, \mathrm{g}} \text { and } \mathrm{Y}_{\mathrm{A}, \mathrm{s}}= & \text { Mole fraction of component } \mathrm{A} \text { in gas } \\
& \text { phase and solid phase, respectively } \\
\mathrm{V}_{\mathrm{Ai}}= & \text { Effectiveness factor of species } \mathrm{A} \text { in } \\
& \text { reaction } \mathrm{i}
\end{array}
$$


$\mathrm{R}_{\mathrm{i}, \mathrm{s}}$

$$
\begin{aligned}
= & \text { Rate expression of reaction } \mathrm{i} \text { (in solid } \\
& \text { phase) in mol sec } \mathrm{m}^{-1} \mathrm{~m}^{-2}
\end{aligned}
$$

Energy equations: For energy balance, enthalpy flowed in the gas phase without chemical reaction and heat convection can transfer from the gas phase to the solid phase. In the solid phase, chemical reaction occurred and heat convection can also transfer from the solid phase to the gas phase. Additionally, heat conduction can occur in the solid phase. These led to internal energy changes in the solid phase. The energy balance in the gas and solid phases can be expressed by Eq. 3 and 4, respectively (Hayes and Kolaczkowski, 1997):

$$
\begin{aligned}
& -\rho_{\mathrm{g}} \mathrm{C}_{\mathrm{pg}} \mathrm{v}_{\mathrm{m}} \frac{\mathrm{dT}}{\mathrm{dx}}+\frac{4}{\mathrm{D}_{\mathrm{H}}} \mathrm{h}\left(\mathrm{T}_{\mathrm{s}}-\mathrm{T}_{\mathrm{g}}\right)=0 \\
& \frac{\partial}{\partial \mathrm{x}}\left(\mathrm{k}_{\mathrm{s}} \delta_{\mathrm{w}} \frac{\partial \mathrm{T}_{\mathrm{s}}}{\partial \mathrm{x}}\right)-\mathrm{h}\left(\mathrm{T}_{\mathrm{s}}-\mathrm{T}_{\mathrm{g}}\right)-\sum_{\mathrm{i}} \Delta \mathrm{H}_{\mathrm{R}, \mathrm{i}} \mathrm{R}_{\mathrm{i}, \mathrm{s}}=\delta_{\mathrm{w}} \rho_{\mathrm{s}} \mathrm{C}_{\mathrm{ps}} \frac{\partial \mathrm{T}_{\mathrm{s}}}{\partial \mathrm{t}}
\end{aligned}
$$

Where:

$\rho_{\mathrm{g}}$ and $\rho_{\mathrm{s}}=$ Gas density and solid phase density, respectively in $\mathrm{kg} \mathrm{m}^{-3}$

$\mathrm{C}_{\mathrm{pg}}$ and $\mathrm{C}_{\mathrm{ps}}=$ Constant pressure heat capacity in gas and solid phase, respectively in $\mathrm{J} \mathrm{kg}^{-1}$ $\mathrm{K}^{-1}$

$\mathrm{v}_{\mathrm{m}}=$ is mean mass average velocity in $\mathrm{msec}^{-1}$

$\mathrm{h}=$ Heat transfer coefficient in $\mathrm{W} \mathrm{m} \mathrm{m}^{-2}$ $\mathrm{K}^{-1}$

$\mathrm{T}_{\mathrm{g}}$ and $\mathrm{T}_{\mathrm{s}}=$ Temperatures in gas and solid phases, respectively in $\mathrm{K}$

$\mathrm{k}_{\mathrm{s}} \quad=\quad$ Solid phase thermal conductivity in $\mathrm{W}$ $\mathrm{m}^{-1} \mathrm{~K}^{-1}$

$\delta_{\mathrm{w}} \quad=$ Reactor wall thickness in $\mathrm{m}$ and $\Delta \mathrm{H}_{\mathrm{R}, \mathrm{i}}$ is enthalpy of reaction $\mathrm{i}$ in $\mathrm{J} \mathrm{mol}^{-1}$

Chemical reactions: The chemical reactions taken place over the catalyst (solid phase) used in this study were based on lumped kinetic reactions extracted from Voltz et al. (1973) and Matthess et al. (2001). Carbon monoxide can oxidize to yield carbon dioxide by Eq. 5 with the rate expression Eq. 8. Propylene $\left(\mathrm{C}_{3} \mathrm{H}_{6}\right)$ represented unburned hydrocarbon in the exhaust gas can also oxidize to yield carbon monoxide and water vapor by Eq. 6 with the rate expression Eq. 9. Propylene can reduce nitric oxide by Eq. 7 with the rate expression Eq. 10. The function $\mathrm{F}\left(\mathrm{Y}, \mathrm{T}_{\mathrm{s}}\right)$ in Eq. 8-10 can be calculated using Eq. 11:

$$
\begin{aligned}
& \mathrm{CO}+\frac{1}{2} \mathrm{O}_{2} \rightarrow \mathrm{CO}_{2} \\
& \frac{1}{3} \mathrm{C}_{3} \mathrm{H}_{6}+\frac{3}{2} \mathrm{O}_{2} \rightarrow \mathrm{CO}_{2}+\mathrm{H}_{2} \mathrm{O}
\end{aligned}
$$

$$
\frac{1}{3} \mathrm{C}_{3} \mathrm{H}_{6}+3 \mathrm{NO} \rightarrow \mathrm{CO}_{2}+\frac{3}{2} \mathrm{~N}_{2}+\mathrm{H}_{2} \mathrm{O}
$$

The rate expressions:

$$
\begin{aligned}
\mathrm{R}_{1, \mathrm{~s}} & =\frac{\mathrm{k}_{1} \mathrm{e}^{\left(-\mathrm{E}_{\mathrm{a} 1} / \tilde{R}_{\mathrm{s}}\right)} \mathrm{Y}_{\mathrm{CO}} \mathrm{Y}_{\mathrm{O}_{2}}}{\mathrm{~F}\left(\mathrm{Y}, \mathrm{T}_{\mathrm{s}}\right)} \\
\mathrm{R}_{2, \mathrm{~s}} & =\frac{\mathrm{k}_{2} \mathrm{e}^{\left(-\mathrm{E}_{\mathrm{a} 2} / \tilde{\mathrm{R}} \mathrm{T}_{\mathrm{s}}\right)} \mathrm{Y}_{\mathrm{C}_{3} \mathrm{H}_{6}} \mathrm{Y}_{\mathrm{O}_{2}}}{\mathrm{~F}\left(\mathrm{Y}, \mathrm{T}_{\mathrm{s}}\right)} \\
\mathrm{R}_{3, \mathrm{~s}} & =\frac{\mathrm{k}_{3} \mathrm{e}^{\left(-\mathrm{E}_{\mathrm{a} 3} / \tilde{\mathrm{R}} \mathrm{T}_{\mathrm{s}}\right)} \mathrm{Y}_{\mathrm{C}_{3} \mathrm{H}_{6}} \mathrm{Y}_{\mathrm{NO}}}{\mathrm{F}\left(\mathrm{Y}, \mathrm{T}_{\mathrm{s}}\right)}
\end{aligned}
$$

Where:

$$
\begin{aligned}
& \mathrm{F}\left(\mathrm{Y}, \mathrm{T}_{\mathrm{s}}\right)=\mathrm{T}_{\mathrm{s}}\left(1+\mathrm{k}_{\mathrm{A} 1} \mathrm{Y}_{\mathrm{CO}}+\mathrm{k}_{\mathrm{A} 2} \mathrm{Y}_{\mathrm{C}_{3} \mathrm{H}_{6}}\right)^{2} \times \\
& \left(1+\mathrm{k}_{\mathrm{A} 3} \mathrm{Y}_{\mathrm{CO}}^{2} \mathrm{Y}_{\mathrm{C}_{3} \mathrm{H}_{6}}^{2}\right)\left(1+\mathrm{k}_{\mathrm{A} 4} \mathrm{Y}_{\mathrm{NO}}^{0.7}\right) \mathrm{K}
\end{aligned}
$$

The pre-exponent factors, activation energy and other constants are listed in Table 1.

Constants and variables: The constant pressure heat capacity $\left(\mathrm{C}_{\mathrm{pg}}\right)$ of the gas phase $\left(\mathrm{J} \mathrm{kg}^{-1} \mathrm{~K}^{-1}\right)$ was dependent on gas temperature and mole fraction of species $\mathrm{i}$ as expressed by Eq. 12 with the constants numerated in Table 2:

$$
\mathrm{C}_{\mathrm{pg}}=\frac{1000}{\mathrm{M}_{\mathrm{g}}} \sum_{\mathrm{i}}\left[\left(\mathrm{m}_{\mathrm{i}, \mathrm{g}}+\mathrm{n}_{\mathrm{i}, \mathrm{g}} \mathrm{T}_{\mathrm{g}}+\mathrm{q}_{\mathrm{i}, \mathrm{g}} \mathrm{T}_{\mathrm{g}}^{2}+\mathrm{r}_{\mathrm{i}}, \mathrm{gT}_{\mathrm{g}}^{3}\right) \mathrm{Y}_{\mathrm{i}, \mathrm{g}}\right]
$$

Table 1: Pre-exponent factors, activation energy and constants for the rate expressions (Voltz et al., 1973; Matthess et al., 2001)

\begin{tabular}{lll}
\hline Item & Unit & Values \\
\hline Pre-exponent factor & $\mathrm{mol} \mathrm{K} \mathrm{sec}^{-1} \mathrm{~m}^{-2}$ & $\mathrm{k}_{1}=6.44 \times 10^{16}$ \\
& & $\mathrm{k}_{2}=1.51 \times 10^{15}$ \\
& & $\mathrm{k}_{3}=7.24 \times 10^{17}$ \\
Activation energy & $\mathrm{J} \mathrm{mol}^{-1 \mathrm{a}}$ & $\mathrm{E}^{\mathrm{a} 1}=119300$ \\
& & $\mathrm{E}_{\mathrm{a} 1}^{\mathrm{a} 1}=85600$ \\
& & $\mathrm{k}_{\mathrm{A}}=65.5 \mathrm{e}^{96 / \mathrm{Ts}}$ \\
& & $\mathrm{k}_{\mathrm{A} 2}=2080 \mathrm{e}^{361 / \mathrm{Ts}}$ \\
& & $\mathrm{k}_{\mathrm{A} 3}=3.98 \mathrm{e}^{11611 / \mathrm{Ts}}$ \\
& & $\mathrm{k}_{\mathrm{A} 4}=4.79 \times 10^{5} \mathrm{e}^{-3733 / \mathrm{Ts}}$ \\
\hline
\end{tabular}

Table 2 Constants for the gas heat capacity (Heywood, 1988)

\begin{tabular}{lrrrr}
\hline Species & \multicolumn{1}{c}{$\mathrm{m}$} & \multicolumn{1}{c}{$\mathrm{n}$} & \multicolumn{1}{c}{$\mathrm{q}$} & $\mathrm{r}$ \\
\hline $\mathrm{NO}$ & 22.29 & $-0.09 \mathrm{E}-2$ & $0.97 \mathrm{E}-5$ & $-4.18 \mathrm{E}-9$ \\
$\mathrm{C}_{3} \mathrm{H}_{6}$ & 3.15 & $23.79 \mathrm{E}-2$ & $-12.15 \mathrm{E}-5$ & $24.58 \mathrm{E}-9$ \\
$\mathrm{CO}$ & 28.11 & $0.16 \mathrm{E}-2$ & $0.53 \mathrm{E}-5$ & $-2.21 \mathrm{E}-9$ \\
$\mathrm{O}_{2}$ & 25.44 & $1.51 \mathrm{E}-2$ & $-0.71 \mathrm{E}-5$ & $1.31 \mathrm{E}-9$ \\
$\mathrm{CO}_{2}$ & 22.22 & $5.97 \mathrm{E}-2$ & $-3.49 \mathrm{E}-5$ & $7.45 \mathrm{E}-9$ \\
$\mathrm{H}_{2} \mathrm{O}$ & 32.19 & $0.19 \mathrm{E}-2$ & $1.05 \mathrm{E}-5$ & $-3.58 \mathrm{E}-9$ \\
$\mathrm{~N}_{2}$ & 28.85 & $-0.15 \mathrm{E}-2$ & $0.80 \mathrm{E}-5$ & $-2.86 \mathrm{E}-9$ \\
\hline
\end{tabular}

Table 3: Constants for the enthalpy of reactions (Heywood, 1988)

\begin{tabular}{lcclll}
\hline Reaction & $\mathrm{a}$ & $\mathrm{b}$ & $\mathrm{c}$ & $\mathrm{d}$ & $\mathrm{f}$ \\
\hline $\mathrm{R}_{1}$ & -279587 & -18.61 & 0.0200 & $-1.22 \mathrm{E}-5$ & $2.25 \mathrm{E}-09$ \\
$\mathrm{R}_{2}$ & -645558 & 15.20 & -0.0200 & $8.94 \mathrm{E}-6$ & $-1.57 \mathrm{E}-10$ \\
$\mathrm{R}_{3}$ & -915767 & 8.76 & -0.0086 & $-3.23 \mathrm{E}-7$ & $9.78 \mathrm{E}-10$ \\
\hline
\end{tabular}


Am. J. Applied Sci., 9 (8): 1225-1231, 2012

Table 4: constants and variables (Hayes and Kolaczkowski, 1999; Matthess et al., 2001)

\begin{tabular}{|c|c|c|}
\hline Item & Unit & Values \\
\hline Gas density & $\mathrm{kg} \mathrm{m}^{3}$ & $\rho_{\mathrm{g}}=\frac{\mathrm{PM}_{\mathrm{g}}}{\tilde{\mathrm{R}} \mathrm{T}_{\mathrm{g}}}$ \\
\hline Solid density & $\mathrm{kg} \mathrm{m}^{3}$ & $\rho_{\mathrm{s}}=1517$ \\
\hline Thermal conductivity of the gas & $\mathrm{W} \mathrm{m}^{-1} \mathrm{~K}^{-1}$ & $\mathrm{k}_{\mathrm{g}}=1.679_{\mathrm{g}}=\times 10^{-2}+5.073 \times 10^{-5} \mathrm{~T}_{\mathrm{g}}$ \\
\hline Thermal conductivity of the solid & $\mathrm{W} \mathrm{m} \mathrm{K}^{-1} \mathrm{~K}^{-1}$ & $\mathrm{k}_{\mathrm{s}}=0.9558-2.09 \times 10^{-4} \mathrm{~T}_{\mathrm{s}}$ \\
\hline Gas concentration & $\mathrm{mol} \cdot \mathrm{m}^{-3}$ & $\mathrm{C}_{\mathrm{g}}=\frac{\mathrm{P}}{\tilde{\mathrm{R}} \mathrm{T}_{\mathrm{g}}}$ \\
\hline Molecular weight of the gas & $\mathrm{kg} \cdot \mathrm{kmol}^{-1}$ & $\mathrm{M}_{\mathrm{g}}=\sum_{\mathrm{i}} \mathrm{M}_{\mathrm{i}} \mathrm{Y}_{\mathrm{i}}$ \\
\hline Heat transfer coefficient & $\mathrm{W} \mathrm{m}{ }^{-2} \mathrm{~K}^{-1}$ & $\mathrm{~h}=\frac{\mathrm{Nu} \cdot \mathrm{k}_{\mathrm{g}}}{\mathrm{D}_{\mathrm{H}}}$ \\
\hline Mass transfer coefficient & $\mathrm{m} \mathrm{sec}^{-1}$ & $\mathrm{k}_{\mathrm{m}}=\frac{\mathrm{Sh} \cdot \mathrm{D}_{\mathrm{A}, \mathrm{B}}}{\mathrm{D}_{\mathrm{H}}}$ \\
\hline Nusselt number & - & $\mathrm{Nu}=3.66$ \\
\hline Sherwood number & - & $\mathrm{Sh}=3.66$ \\
\hline Hydraulic diameter of monolith channel & $\mathrm{m}$ & $\mathrm{D}_{\mathrm{H}}=1.09 \times 10^{-3}$ \\
\hline Wall thickness & $\mathrm{m}$ & $\delta_{\mathrm{w}}=0.1388 \times 10^{-3}$ \\
\hline Heat capacity of the solid & $\mathrm{J} \mathrm{kg}^{-1} \mathrm{~K}^{-1}$ & $\mathrm{C}_{\mathrm{ps}}=984+0.226 \mathrm{~T}_{\mathrm{s}}$ \\
\hline
\end{tabular}

The standard enthalpy of reaction $\mathrm{i}\left(\Delta \mathrm{H}_{\mathrm{R}, \mathrm{i}}^{\circ}\right)$ in $\mathrm{J} \cdot \mathrm{mol}^{-1}$ was a function of temperature of the solid phase as given by Eq. 13 with the constants numerated in Table 3:

$$
\Delta \mathrm{H}_{\mathrm{R}, \mathrm{i}}^{\circ}=\mathrm{a}+\mathrm{bT}_{\mathrm{s}}+\mathrm{cT}_{\mathrm{s}}^{2}+\mathrm{dT}_{\mathrm{s}}^{3}+\mathrm{fT}_{\mathrm{s}}^{4}
$$

Other constants and variables used in the species balance and the energy balance in the gas and solid phases, extracted from Hayes and Kolaczkowski (1999) and Matthess et al. (2001) are given in Table 4.

Diffusion coefficient for species A diffusing into a binary mixture of $A$ and $B\left(D_{A, B}\right)$ in $\mathrm{m}^{2} \sec ^{-1}$ can be written as Eq. 14 (Fuller et al., 1966):

$$
\mathrm{D}_{\mathrm{A}, \mathrm{B}}=\frac{1.013 \times 10^{-2} \mathrm{~T}^{1.75} \sqrt{\frac{1}{\mathrm{M}_{\mathrm{A}}}+\frac{1}{\mathrm{M}_{\mathrm{B}}}}}{\mathrm{P}\left[\left(\sum \mathrm{v}\right)_{\mathrm{A}}^{1 / 3}+\left(\sum \mathrm{v}\right)_{\mathrm{B}}^{1 / 3}\right]^{2}}
$$

And diffusion coefficient for species A diffusing into a multi-component mixture $\left(\mathrm{D}_{\mathrm{A}, \mathrm{mix}}\right)$ in $\mathrm{m}^{2} \mathrm{sec}^{-1}$ can be expressed by Eq. 15 .

$D_{A, \operatorname{mix}}=\left(\sum_{\substack{j=1 \\ j \neq i}}^{n} \frac{Y_{i j}}{D_{i j}}\right)^{-1}$

Gas conversion percentage can be calculated using Eq. 16:

$\operatorname{Gas}$ Conversion $(\%)=\frac{Y_{g, \text { in }}-Y_{g, \text { out }}}{Y_{g, \text { in }}} \times 100$
Numerical algorithm: The gas phase species balance Eq. 1 was rearranged in the form of finite difference scheme, appearred in Eq. 17 and 18. For NO emission:

$\mathrm{v}_{\mathrm{m}}^{*}\left[\frac{\mathrm{Y}_{\mathrm{NO}, \mathrm{g}, \mathrm{i}}-\mathrm{Y}_{\mathrm{NO}, \mathrm{g}, \mathrm{i}-1}}{\Delta \mathrm{x}}\right]+\frac{4}{\mathrm{D}_{\mathrm{H}}} \mathrm{k}_{\mathrm{m}, \mathrm{NO}}\left(\mathrm{Y}_{\mathrm{NO}, \mathrm{g}, \mathrm{i}}-\mathrm{Y}_{\mathrm{NO}, \mathrm{s}, \mathrm{i}}\right)=0$

Thus:

$$
\mathrm{Y}_{\mathrm{NO}, \mathrm{g}, \mathrm{i}}=\frac{\mathrm{Y}_{\mathrm{NO}, \mathrm{g}, \mathrm{i}-1}}{\left[1+\frac{4 \mathrm{k}_{\mathrm{m}, \mathrm{NO}} \Delta \mathrm{x}}{\mathrm{D}_{\mathrm{H}} \mathrm{v}_{\mathrm{m}}^{*}}\right]}+\frac{\mathrm{Y}_{\mathrm{NO}, \mathrm{s}, \mathrm{i}}}{\left[1+\frac{\mathrm{D}_{\mathrm{H}} \mathrm{v}_{\mathrm{m}}^{*}}{4 \mathrm{k}_{\mathrm{m}, \mathrm{NO}} \Delta \mathrm{x}}\right]}
$$

And the other six species can be derived in the same manner, generating a set of equations.

The solid phase species balance Eq. 2 was rearranged in the form of finite difference scheme, expressed in Eq. 19-32. For all species:

$$
\begin{aligned}
& \mathrm{k}_{\mathrm{m}, \mathrm{NO}} \mathrm{C}_{\mathrm{g}}\left(\mathrm{Y}_{\mathrm{NO}, \mathrm{g}}-\mathrm{Y}_{\mathrm{NO}, \mathrm{s}}\right)-3 \mathrm{R}_{3}=0 \\
& \mathrm{k}_{\mathrm{m}, \mathrm{C}_{3} \mathrm{H}_{6}} \mathrm{C}_{\mathrm{g}}\left(\mathrm{Y}_{\mathrm{C}_{3} \mathrm{H}_{6}, \mathrm{~g}}-\mathrm{Y}_{\mathrm{C}_{3} \mathrm{H}_{6}, \mathrm{~s}}\right)-\frac{1}{3} \mathrm{R}_{2}-\frac{1}{3} \mathrm{R}_{3}=0 \\
& \mathrm{k}_{\mathrm{m}, \mathrm{CO}} \mathrm{C}_{\mathrm{g}}\left(\mathrm{Y}_{\mathrm{CO}, \mathrm{g}}-\mathrm{Y}_{\mathrm{CO}, \mathrm{s}}\right)-\mathrm{R}_{1}=0
\end{aligned}
$$

$\mathrm{k}_{\mathrm{m}, \mathrm{O}_{2}} \mathrm{C}_{\mathrm{g}}\left(\mathrm{Y}_{\mathrm{O}_{2}, \mathrm{~g}}-\mathrm{Y}_{\mathrm{O}_{2}, \mathrm{~s}}\right)-\frac{1}{2} \mathrm{R}_{1}-\frac{1}{2} \mathrm{R}_{2}=0$

$\mathrm{k}_{\mathrm{m}, \mathrm{CO}_{2}} \mathrm{C}_{\mathrm{g}}\left(\mathrm{Y}_{\mathrm{CO}_{2}, \mathrm{~g}}-\mathrm{Y}_{\mathrm{CO}_{2}, \mathrm{~s}}\right)+\mathrm{R}_{1}+\mathrm{R}_{2}+\mathrm{R}_{3}=0$ 


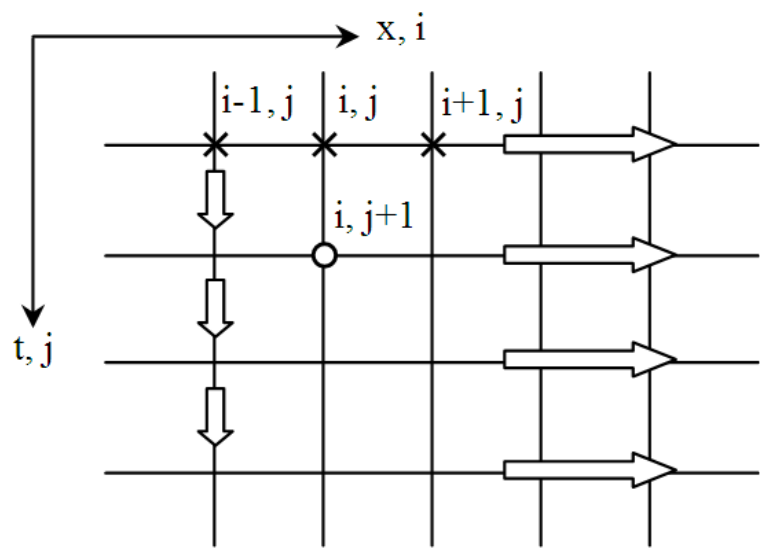

Fig. 3: Finite difference approximation flowchart $(0=$ unknown value, $x=$ known values)

Table 5: Initial conditions of the mole fraction

\begin{tabular}{ll}
\hline Species & Mole fraction $\left(\mathrm{Y}_{\mathrm{i}}\right)$ \\
\hline $\mathrm{NO}$ & 0.0030 \\
$\mathrm{C}_{3} \mathrm{H}_{6}$ & 0.0024 \\
$\mathrm{CO}$ & 0.0010 \\
$\mathrm{O}_{2}$ & 0.0100 \\
$\mathrm{CO}_{2}$ & 0.1000 \\
$\mathrm{H}_{2} \mathrm{O}$ & 0.1000 \\
$\mathrm{~N}_{2}$ & Balance \\
\hline
\end{tabular}

$\mathrm{k}_{\mathrm{m}, \mathrm{H}_{2} \mathrm{O}} \mathrm{C}_{\mathrm{g}}\left(\mathrm{Y}_{\mathrm{H}_{2} \mathrm{O}, \mathrm{g}}-\mathrm{Y}_{\mathrm{H}_{2} \mathrm{O}, \mathrm{s}}\right)+\mathrm{R}_{2}+\mathrm{R}_{3}=0$

$\mathrm{k}_{\mathrm{m}, \mathrm{N}_{2}} \mathrm{C}_{\mathrm{g}}\left(\mathrm{Y}_{\mathrm{N}_{2}, \mathrm{~g}}-\mathrm{Y}_{\mathrm{N}_{2}, \mathrm{~s}}\right)+\frac{3}{2} \mathrm{R}_{3}=0$

And, thus:

$$
\begin{aligned}
& \mathrm{Y}_{\mathrm{NO}, \mathrm{s}, \mathrm{i}}=\mathrm{Y}_{\mathrm{NO}, \mathrm{g}, \mathrm{i}-1}+\frac{\left(-3 \mathrm{R}_{3}\right)}{\mathrm{k}_{\mathrm{m}, \mathrm{NO}} \mathrm{C}_{\mathrm{g}}}\left[\frac{4 \mathrm{k}_{\mathrm{m}, \mathrm{NO}} \Delta \mathrm{x}}{\mathrm{D}_{\mathrm{H}} \mathrm{v}_{\mathrm{m}}^{*}}+1\right] \\
& \mathrm{Y}_{\mathrm{C}_{3} \mathrm{H}_{6}, \mathrm{~s}, \mathrm{i}}=\mathrm{Y}_{\mathrm{C}_{3} \mathrm{H}_{6}, \mathrm{~g}, \mathrm{i}-\mathrm{i}}+\frac{\left(-\mathrm{R}_{2}-\mathrm{R}_{3}\right)}{\mathrm{k}_{\mathrm{m}, \mathrm{C}_{3} \mathrm{H}_{6}} \mathrm{C}_{\mathrm{g}}}\left[\frac{4 \mathrm{k}_{\mathrm{m}, \mathrm{C}_{3} \mathrm{H}_{6}} \Delta \mathrm{x}}{\mathrm{D}_{\mathrm{H}_{\mathrm{m}}} \mathrm{v}_{\mathrm{m}}^{*}}+1\right] \\
& \mathrm{Y}_{\mathrm{CO}, \mathrm{s}, \mathrm{i}}=\mathrm{Y}_{\mathrm{CO}, \mathrm{g}, \mathrm{i}-1}+\frac{\left(-\mathrm{R}_{1}\right)}{\mathrm{k}_{\mathrm{m}, \mathrm{CO}} \mathrm{C}_{\mathrm{g}}}\left[\frac{4 \mathrm{k}_{\mathrm{m}, \mathrm{CO}} \Delta \mathrm{x}}{\mathrm{D}_{\mathrm{H}} \mathrm{v}_{\mathrm{m}}^{*}}+1\right] \\
& \mathrm{Y}_{\mathrm{O}_{2}, \mathrm{~s}, \mathrm{i}}=\mathrm{Y}_{\mathrm{O}_{2}, \mathrm{~g}, \mathrm{i}-1}+\frac{\left(-0.5 \mathrm{R}_{1}-1.5 \mathrm{R}_{2}\right)}{\mathrm{k}_{\mathrm{m}, \mathrm{O}_{2}} \mathrm{C}_{\mathrm{g}}}\left[\frac{4 \mathrm{k}_{\mathrm{m}, \mathrm{O}_{2}} \Delta \mathrm{x}}{\mathrm{D}_{\mathrm{H}} \mathrm{v}_{\mathrm{m}}^{*}}+1\right] \\
& \mathrm{Y}_{\mathrm{CO}_{2}, \mathrm{~s}, \mathrm{i}}=\mathrm{Y}_{\mathrm{CO}_{2}, \mathrm{~g}, \mathrm{i}-1}+\frac{\left(\mathrm{R}_{1}+\mathrm{R}_{2}+\mathrm{R}_{3}\right)}{\mathrm{k}_{\mathrm{m}, \mathrm{CO}_{2}} \mathrm{C}_{\mathrm{g}}}\left[\frac{4 \mathrm{k}_{\mathrm{m}, \mathrm{CO}} \Delta \mathrm{x}}{\mathrm{D}_{\mathrm{H}} \mathrm{v}_{\mathrm{m}}^{*}}+1\right] \\
& \mathrm{Y}_{\mathrm{H}_{2} \mathrm{O}, \mathrm{s}, \mathrm{i}}=\mathrm{Y}_{\mathrm{H}_{2} \mathrm{O}, \mathrm{g}, \mathrm{i}-1}+\frac{\left(\mathrm{R}_{2}+\mathrm{R}_{3}\right)}{\mathrm{k}_{\mathrm{m}, \mathrm{H}_{2} \mathrm{O}} \mathrm{C}_{\mathrm{g}}}\left[\frac{4 \mathrm{k}_{\mathrm{m}, \mathrm{H}_{2} \mathrm{O}} \Delta \mathrm{x}}{\mathrm{D}_{\mathrm{H}} \mathrm{v}_{\mathrm{m}}^{*}}+1\right]
\end{aligned}
$$

$$
\mathrm{Y}_{\mathrm{N}_{2}, \mathrm{~s}, \mathrm{i}}=\mathrm{Y}_{\mathrm{N}_{2}, \mathrm{~g}, \mathrm{i}-1}+\frac{\left(1.5 \mathrm{R}_{3}\right)}{\mathrm{k}_{\mathrm{m}, \mathrm{N}_{2}} \mathrm{C}_{\mathrm{g}}}\left[\frac{4 \mathrm{k}_{\mathrm{m}, \mathrm{N}_{2}} \Delta \mathrm{x}}{\mathrm{D}_{\mathrm{H}} \mathrm{v}_{\mathrm{m}}^{*}}+1\right]
$$

The gas phase energy balance Eq. 3 was rearranged in the form of finite difference scheme, shown in Eq. 33 and 34:

$$
\mathrm{v}_{\mathrm{m}} \rho_{\mathrm{g}} \mathrm{C}_{\mathrm{pg}}\left[\frac{\mathrm{T}_{\mathrm{g}, \mathrm{i}}-\mathrm{T}_{\mathrm{g}, \mathrm{i}-1}}{\Delta \mathrm{x}}\right]+\frac{4}{\mathrm{D}_{\mathrm{H}}} \mathrm{h}\left(\mathrm{T}_{\mathrm{g}, \mathrm{i}}-\mathrm{T}_{\mathrm{s}, \mathrm{i}}\right)=0
$$

And, thus:

$$
\mathrm{T}_{\mathrm{g}, \mathrm{i}}=\left[\frac{\mathrm{T}_{\mathrm{g}, \mathrm{i}-1}}{1+\frac{4 \mathrm{~h} \Delta \mathrm{x}}{\mathrm{D}_{\mathrm{H}} \mathrm{v}_{\mathrm{m}} \rho_{\mathrm{g}} \mathrm{C}_{\mathrm{pg}}}}\right]+\left[\frac{\mathrm{T}_{\mathrm{s,i}}}{1+\frac{\mathrm{D}_{\mathrm{H}} \mathrm{v}_{\mathrm{m}} \rho_{\mathrm{g}} \mathrm{C}_{\mathrm{pg}}}{4 \mathrm{~h} \Delta \mathrm{x}}}\right]
$$

The solid phase energy balance Eq. 4 was rearranged in the form of finite difference scheme as in Eq. 35 and 36:

$$
\begin{aligned}
& \mathrm{k}_{\mathrm{s}} \delta_{\mathrm{w}}\left[\frac{\mathrm{T}_{\mathrm{s}, \mathrm{i}+1}^{\mathrm{t}}-2 \mathrm{~T}_{\mathrm{s}, \mathrm{j}}^{\mathrm{t}}+\mathrm{T}_{\mathrm{s}, \mathrm{j}-1}^{\mathrm{t}}}{(\Delta \mathrm{x})^{2}}\right]-\mathrm{h}\left(\mathrm{T}_{\mathrm{s}, \mathrm{i}}-\mathrm{T}_{\mathrm{g}, \mathrm{i}}\right)-\Delta \mathrm{H}_{\mathrm{R} 1} \mathrm{R}_{\mathrm{ls}} \\
& -\Delta \mathrm{H}_{\mathrm{R} 2} \mathrm{R}_{2 \mathrm{~s}}-\Delta \mathrm{H}_{\mathrm{R} 3} \mathrm{R}_{3 \mathrm{~s}}=\delta_{\mathrm{w}} \rho_{\mathrm{s}} \mathrm{C}_{\mathrm{ps}}\left[\frac{\mathrm{T}_{\mathrm{s}, \mathrm{i}}^{\mathrm{t}+\Delta \mathrm{t}}-\mathrm{T}_{\mathrm{s}, \mathrm{i}}^{\mathrm{t}}}{\Delta \mathrm{t}}\right]
\end{aligned}
$$

And, thus:

$$
\mathrm{T}_{\mathrm{s}, \mathrm{i}}^{\mathrm{t}+\Delta t}=\mathrm{T}_{\mathrm{s}, \mathrm{i}}^{\mathrm{t}}+\frac{\Delta \mathrm{t}}{\delta_{\mathrm{w}} \rho_{\mathrm{s}} \mathrm{C}_{\mathrm{ps}}}\left[\begin{array}{l}
\mathrm{k}_{\mathrm{s}} \delta_{\mathrm{w}}\left(\frac{\mathrm{T}_{\mathrm{s}, \mathrm{t}+1}^{\mathrm{t}}-2 \mathrm{~T}_{\mathrm{s}, \mathrm{i}}^{\mathrm{t}}+\mathrm{T}_{\mathrm{s}, \mathrm{i}-1}^{\mathrm{t}}}{(\Delta \mathrm{x})^{2}}\right) \\
-\mathrm{h}\left(\mathrm{T}_{\mathrm{s}, \mathrm{i}}-\mathrm{T}_{\mathrm{g}, \mathrm{i}}\right)- \\
\Delta \mathrm{H}_{\mathrm{R} 1} \mathrm{R}_{1 \mathrm{~s}}-\Delta \mathrm{H}_{\mathrm{R} 2} \mathrm{R}_{2 \mathrm{~s}}-\Delta \mathrm{H}_{\mathrm{R} 3} \mathrm{R}_{3 \mathrm{~s}}
\end{array}\right]
$$

The finite difference scheme with explicit central difference approximation is shown in Fig. 3.

A computer code was generated to solve for mole fraction $\left(\mathrm{Y}_{\mathrm{g}, \mathrm{i}}\right)$ and temperature $\left(\mathrm{T}_{\mathrm{g}, \mathrm{i}}\right)$ over spatial $(\mathrm{x})$ and time (t) domains.

Initial and boundary conditions: The initial condition for temperatures of the gas phase $\left(\mathrm{T}_{\mathrm{gi}}\right)$ was set to a range between 400 and $620 \mathrm{~K}$ while that of the solid phase $\left(\mathrm{T}_{\mathrm{si}}\right)$ was set to $298 \mathrm{~K}$.

For mole fraction, the initial conditions were set to the values shown in Table 5. These values simulated the engine operating on fuel-lean combustion condition.

It was considered that there was no change in solid temperature gradient along the $\mathrm{x}$-axis. Therefore, the boundary condition at both ends of the solid wall $(\mathrm{x}=0$ and $\mathrm{x}=\mathrm{L}$ ) was as Eq. 37:

$\frac{\mathrm{dT}_{\mathrm{s}}}{\mathrm{dx}}=0$ 


\section{RESULTS}

Nitric oxide conversion in initial transient state: The calculation results of NO conversion at the catalyst bed outlet $(\mathrm{x}=0.16 \mathrm{~m})$ with $0.625-\mathrm{m} \mathrm{sec}^{-1}$ gas velocity are shown in Fig. 4. The NO conversion began to increase after the time started. They obviously showed the changes in transient period from 2-12 sec and afterwards, they reached steady state at different values.

Effects of inlet temperature on NO conversion: Inlet gas temperature affected on NO conversion at the catalyst bed outlet $(x=0.16 \mathrm{~m})$ with the gas velocity of $0.625 \mathrm{~m} \mathrm{sec}^{-1}$ as shown in Fig. 5. It was observed that the light-off temperatures were up to $520 \mathrm{~K}$ whereas the NO conversion was nearly zero. The calculation results showed a sudden rise in NO conversion from approximately 550-605 K and decreasing onwards.

Effects of gas velocity on NO conversion: Gas velocity affected on NO conversion at $600-\mathrm{K}$ inlet gas temperature over the catalyst bed is shown in Fig. 6. NO started conversion at the catalyst bed inlet and the NO conversion decreased as the gas velocity increased. The NO increased conversion along the catalyst bed for all the gas velocity.

\section{DISCUSSION}

In Fig. 4, Inlet gas temperature strongly affects on the NO conversion. In the range of inlet gas temperature tested $(560-600 \mathrm{~K})$, it is observed that the lower inlet gas temperature resulted in lesser NO conversion at the same inlet NO concentration and gas velocity.

In Fig. 5, the maximum $\mathrm{NO}$ conversion was $76 \%$ at this numerical test condition $(\mathrm{x}=0.16 \mathrm{~m}$ and gas velocity $=0.625 \mathrm{~m} \mathrm{sec}^{-1}$ ). This generated a working temperature window for $\mathrm{NO}$ conversion simulated by this model with the variables and chemical reaction proposed. The temperature window was validated and compared with the experimental and simulation results from the literature (Matthess et al., 2001). They gave the same trend but differently in values, due to differences in variables and conditions used.

Figure 6 the greater gas velocity resulted in lower NO conversion, due to the shorter residence (contact) time. In contrast, at the lower gas velocity, the gas flow had more times for reaction, resulting higher $\mathrm{NO}$ conversion. However, at very low gas velocity of $0.425 \mathrm{~m} \mathrm{sec}^{-1}$, the NO conversion started to deviate at the middle of the catalyst bed, resulting in lower conversion at the catalyst outlet than expected. This implies that it may not be suitable to operate at gas velocity lower than this as it may result in low NO conversion.

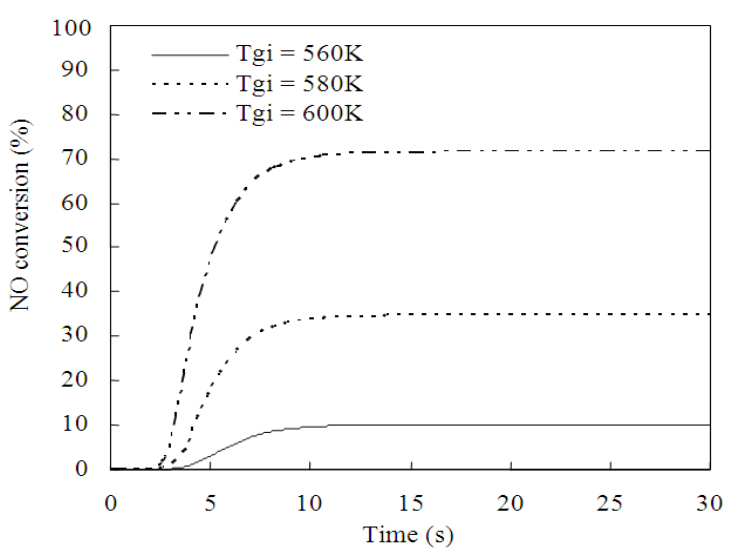

Fig. 4: NO conversion in initial transient state at $\mathrm{x}=$ $0.16 \mathrm{~m}$ and gas velocity $=0.625 \mathrm{~m} \mathrm{sec}^{-1}$

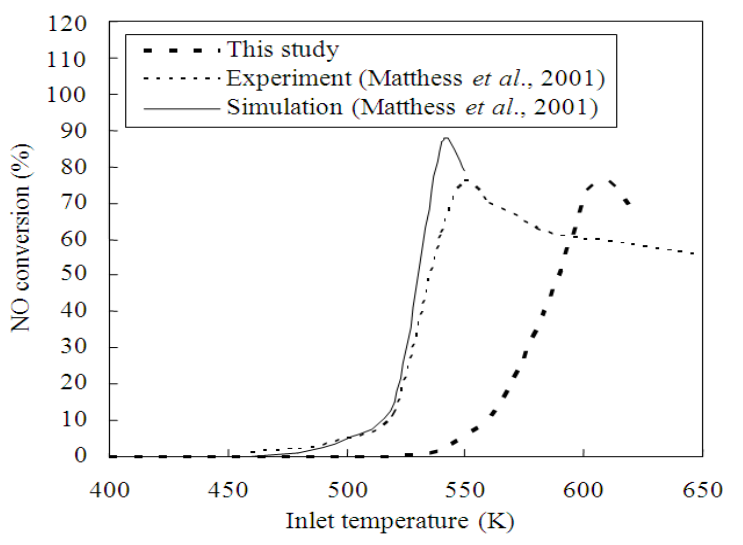

Fig. 5: Inlet gas temperature effects on NO conversion at $\mathrm{x}=0.16 \mathrm{~m}$ and gas velocity $=0.625 \mathrm{~m} \mathrm{sec}^{-1}$

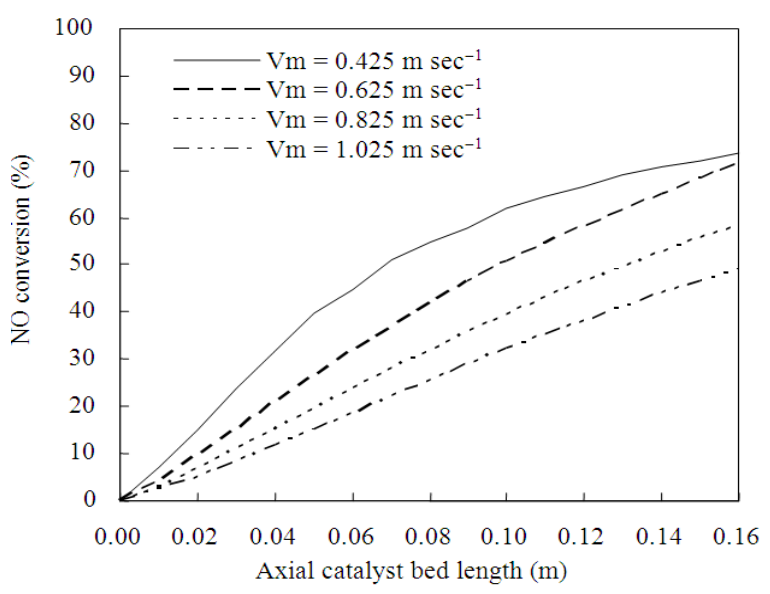

Fig. 6: Gas velocity effects on $\mathrm{NO}$ conversion at $\mathrm{T}_{\mathrm{gi}}=$ $600 \mathrm{~K}$ 


\section{CONCLUSION}

The conversion of NO emission in monolith catalytic converter was numerically studied using heterogeneous combustion in one-dimensional transient model. The analysis on species and energy balances was accomplished and solved by finite difference method. Gas inlet temperature and velocity were found to affect on NO conversion over the time and spatial domains. The calculated working temperature window for NO conversion was in agreement with experimental and simulation results from the literature.

\section{ACKNOWLEDGEMENT}

The researcher acknowledges the Kasetsart University Research and Development Institute (KURDI) and the Kasetsart University Center for Advanced Studies in Industrial Technology under the National Research University (NRU) project for the support to this study.

\section{REFERENCES}

Botas, J.A., M.A. Gutiérrez-Ortiz, M.P. GonzálezMarcos, J.A. González-Marcos and J.R. GonzálezVelasco, 2001. Kinetic considerations of three-way catalysis in automobile exhaust converters. Applied Catal., B: Environ., 32: 243-256. DOI: 10.1016/S0926-3373(01)00144-8

Burch, R. and T.C. Watling, 1997. Kinetics and mechanism of the reduction of $\mathrm{NO}$ by $\mathrm{C}_{3} \mathrm{H}_{8}$ Over $\mathrm{Pt} / \mathrm{Al}_{2} \mathrm{O}_{3}$ under lean-burn conditions. J. Catal., 169: 45-54. DOI: 10.1006/jcat.1997.1686

Fuller, E.N., P.D. Schettler and J.C. Giddings, 1966. New method for prediction of binary gas-phase diffusion coefficients. Ind. Eng. Chem., 58: 18-27. DOI: 10.1021/ie50677a007

Furusawa, T., K. Seshan, J.A. Lercher, L. Lefferts and K.I. Aika, 2002. Selective reduction of $\mathrm{NO}$ to $\mathrm{N}_{2}$ in the presence of oxygen over supported silver catalysts. Applied Catal. B: Environ., 37: 205-216. DOI: 10.1016/S0926-3373(01)00337-X
Hayes, R.E. and S.T. Kolaczkowski, 1997. Introduction to Catalytic Combustion. 1st Edn., Gordon and Breach, Australia, ISBN-10: 9056990926, pp: 681.

Hayes, R.E. and S.T. Kolaczkowski, 1999. A study of nusselt and sherwood numbers in a monolith reactor. Catal. Today, 47: 295-303. DOI: 10.1016/S0920-5861(98)00310-1

Hepburn, J., T. Kenney, J. McKenzie, E. Thanasiu and M. Dearth, 1998. Engine and aftertreatment modeling for gasoline direct injection. SAE Technical Paper. DOI: 10.4271/982596

Heywood, J.B., 1988. Internal Combustion Engine Fundamentals. 1st Edn., McGraw-Hill, New York, ISBN-10: 007028637X, pp: 930.

Holma, T., A. Palmqvist, M. Skoglundh and E. Jobson, 2004. Continuous lean $\mathrm{NO}_{\mathrm{x}}$ reduction with hydrocarbons over dual pore system catalysts. Applied Catal., B: Environ., 48: 95-100. DOI: 10.1016/j.apcatb.2003.09.021

Kolaczkowski, S.T., 1999. Modelling catalytic combustion in monolith reactors - challenges faced. Catal. Today, 47: 209-118. DOI: 10.1016/S0920-5861(98)00301-0

Matthess, N., D. Schweichb, B. Martin and F. Castagna, 2001. From light-off curves to kinetic rate expressions for three-way catalysts. Top. Catal., 16: 119-124. DOI: 10.1023/A:1016695101135

Tischer, S., C. Correa and O. Deutschmann, 2001. Transient three-dimensional simulations of a catalytic combustion monolith using detailed models for heterogeneous and homogeneous reactions and transport phenomena. Catal. Today, 69: 57-62. DOI: 10.1016/S0920-5861(01)00355-8

Voltz, S.E., C.R. Morgan and D. Liederman, 1973. Kinetic study of carbon monoxide and propylene oxidation on platinum catalysts. Ind. Eng. Chem. Prod. Res. Dev., 12: 294-301. DOI: 10.1021/i360048a006 